\title{
LPG GAS DETECTING ROBOT BASED ON IOT
}

\author{
Tanzila Younas $^{1 *}$, Deepak Kumar Mukhi ${ }^{1}$, Mufaddal Saifuddin ${ }^{1}$, S M Faiz Hassan Zaidi ${ }^{1}$, M Mahad Fayyaz ${ }^{1}$, and Hamzah \\ Ahmad Khan ${ }^{1}$ \\ ${ }^{1}$ Mechatronics Department, SZABIST, 100 Clifton,75600, Karachi, Pakistan
}

\begin{abstract}
With new innovations and technologies world besides being facilitated is also subjected to face the threat of major misadventures and disasters. Likewise, Liquefied Petroleum Gas (LPG) is generally used in household for kitchen in general and gas geysers or heaters in winter. Similarly, industries use it for different causes for instance furnace, boiling and get higher production at cheaper rate. Besides, this gas is igneous gas and can cause potential hazard at any time. Hence, in this paper research is made on Internet of Things (IOT) to reduce the chances of such mishaps by presenting a model of microcontroller-based LPG gas detecting and warning system. Furthermore, it explicates the development of self-directed android based mobile device for gas leakage detection that can be installed in all sort of places. Moreover, this device would be containing MQ6 sensor to detect the unwanted outflow and GPS module to locate particular location of leakage what is more Arduino is installed to disseminate information of leakage to different servers like Smartphones, Email and buzzer and shall give display on LCD. Subsequently, it will send the mobile alert to authorized and concerned authorities to take action with in time and there would be buzzer installed. In addition, this device promises automatic working with no need of human interference and assures reliability, efficiency along with cost-effectiveness check-ups of pipeline.
\end{abstract}

\section{Introduction}

World with the landing in scientific century has revealed number of innovations and has brought technological revolution to facilitate human being. Today, technology is nothing but an extension of human being it has penetrated every place and has become a necessity. The man of today cannot imagine his life without these scientific machines rather astonishes how humans in Stone Age managed to survive. Nonetheless, as every picture has both sides bright and dark, similarly, besides these easements from technology it also brings the perils of misadventures. However, owing to daily new researches and ideas new technology is being innovated to surmount the hazards and dangers posed by existing ones[1-2]. This paper specifically aims to reduce the dangers of Liquefied Petroleum Gas (LPG) leakage anywhere be it home, office, industries or supplying pipelines. Furthermore, this paper intends to formulate a device that can detect the leakage with no human interference and lessen the chances of any hazard caused by such leakage by alerting the concerned authorities and authorized persons. [3] Thus, Arduino or Raspberry pi will be installed to control the other mounted circuits or devices that will help in making this objective of decreasing misadventure by gas leakage possible and it will disseminate information regarding leakage. As, the robot will be consisting MQ-6 sensor which detects gas leakage explicitly Methane (CH4), Propane (C3H8), Butane (C4H12). Moreover, this device will directly be connected with GPS module to locate the location of particular leakage in pipeline hence resulting in alarming all the connected devices for this cause. The usage of Internet of Things is mention worthy and pertinent to the purpose of this device in its entirety. For instance, The Internet of Things (IoT) can be labelled as linking routine objects like smart phones, TVs, sensors, buzzer, Email, siren and actuators to the Internet where devices are technically linked together facilitating new forms of communication between things and people and between things themselves.

\section{Literature Review}

Science has been continuously advancing and new studies are incessantly taking place to overcome the gaps left by any technology. Similarly, in 2008, LIU zhen-ya, WANG Zhen-dong and CHEN Rong's, "Intelligent Private Security Caution and Remote Control Framework Built on Single Chip Computer", was published their paper's main focus was centered on, intelligent private burglar caution, crisis sound alert, fire caution, harmful fume spillage inaccessible programmed sound alert and farther control framework, which relies on $89 \mathrm{c} 51$ single chip computer. Moreover, this framework [4] can execute

\footnotetext{
* Corresponding author: tanzila@szabist.edu.pk
} 
a programmed caution, which can make phone call to the police hotline number directly. It can also be a voice alert and caution to be appeared on LCD. This security framework can be utilized in controlling the electrical control remotely through telephone [5].

Besides, In 2008, Chen Peijiang and Jiang Xuehhua's, got their research published named "Design and usage of Inaccessible Observing Framework Based on GSM", and made a point that this research is centered on the remote checking framework, since the remote farther checking framework has added applications a farther observing framework based on SMS through GSM.

Moreover, much work has been done and number of research papers have been written and published on the above mentioned robot and indeed the most recent literature published regarding this Internet of Things (IOT) Centered Gas Leakage Monitoring and Alerting System with MQ-6 Sensor published in 2017 by International Journal of Engineering Development and Research (IJEDR) which highlighted the very concern of possible mishap by leakage of toxic gas leading to loss to human lives[7-8] and property and gives a way-forward to get it away with the invention of leakage detector robot.

\section{Methodology}

The proposed device is delivered using the Raspberry Pi Raspberry Pi would possibly be a computerized PC which may have made and balanced the absolutely extraordinary ways it licenses us to run a range of undertakings and in addition reinforces particular peripherals in various manners with which it grants us to run various activities and besides guide various peripherals which are to be used in the system MQ Sensors are introduced on the motive of the LPG Supply to apprehend the spillage of gas, Once the catch facet is completed it will send an alert message to control adaptable, The message is send to Email or at smart phone. [9] LED is advised whilst gas spillage takes places. The alarming signal is installed with the system. This data is stored in website page using Internet of Things. The entire phenomena taking place at the device can be witnessed by executing a python code and via imparting the required sensors.

\subsection{Control Unit}

A RASPBERRY PI 3 The raspberry pie 3 is used in the robot, which boosts UNIX framework and python language. Multiple gadgets are associated with Raspberry pie to recognize the leakage of gas from pipeline. Further the module having containing for stick Breadboard, session commencement convention, one I/O for Micro-controller. In fact, when unwanted or accidental leakage takes place it immediately alerts the Micro-controller to send the message to the respective authorized person and concerned Gas organization. Its shape seems like that of an ATM $\operatorname{card}[10]$.

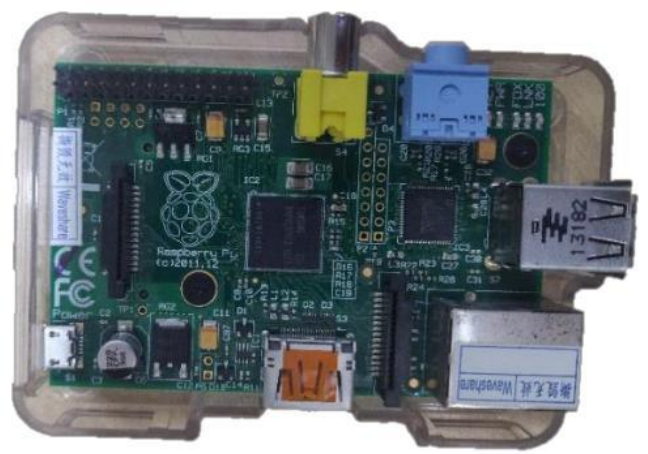

Fig. 1 RASSBERRY PI [11]

This is the MQ6 gas location module which is for the most part utilized for examination of gas leakage in the specific territory of this sensor. It is specifically used to gauge the concentration of gas. It predominantly perceives LPG, coal, Alcohol etc.

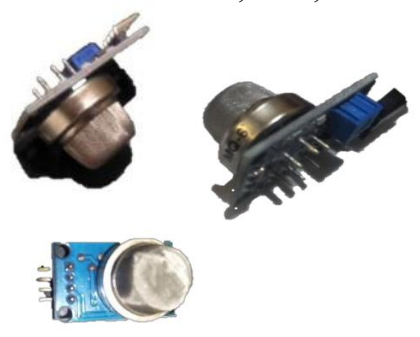

Fig. 2 MQ6 SENSOR [12]

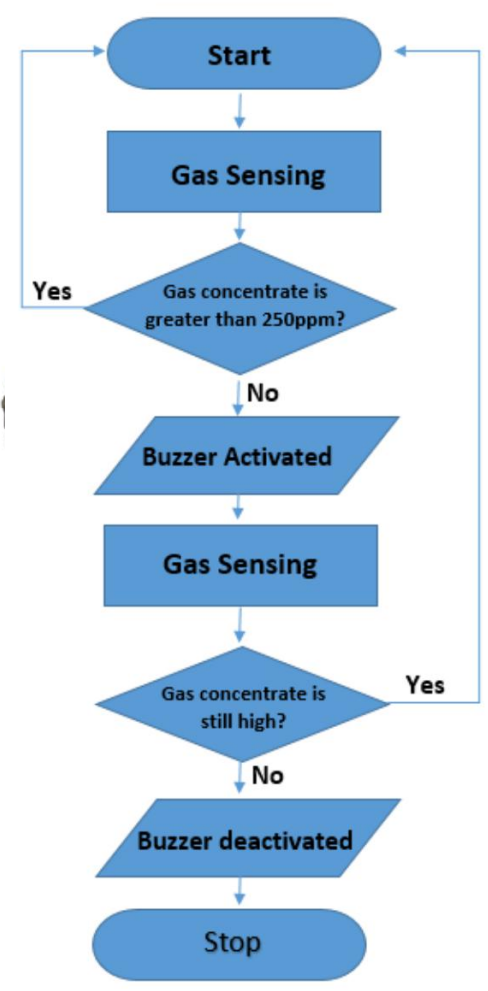

Fig. 3 Flowchart of the system 


\section{CAD Model of the Robot}

The design of the 4 wheel bot with a SS-base plate where the wheels are attached is shown in figure 4. 2 DC gear motors with high load torque provides the movement of robot. Operating voltage of the motors is between $12 \mathrm{~V}$ to $24 \mathrm{~V}$. For smooth and free movement two caster wheels are attached.

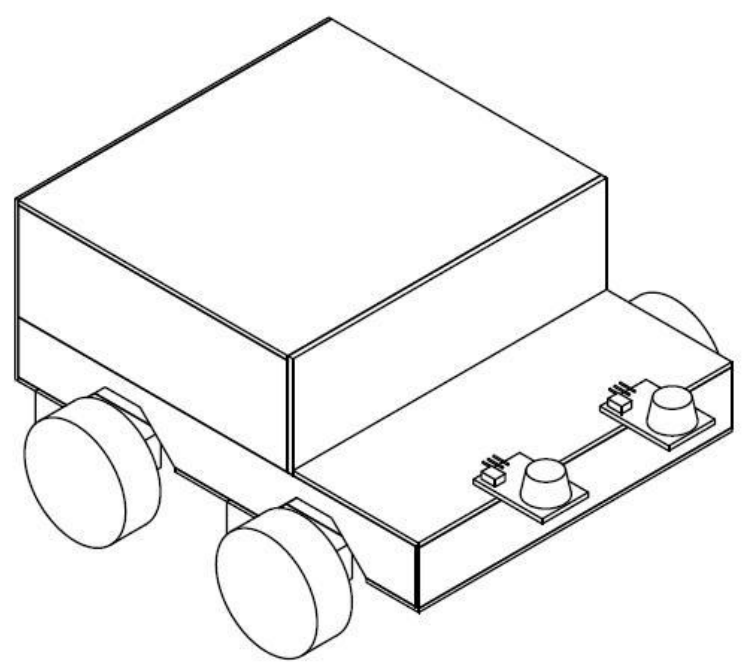

Fig. 4 Gas detecting robot

\section{Discussion}

The gas detecting robot is tested by releasing LPG gas around the robot in the atmosphere. The fully functional gas detecting robot consists of the LPG detecting sensor and control unit to detect this LPG gas in the atmosphere [13].

This simple test was carried out multiple times at different time of the day and night, as at every time there would be different concentration of the air in the atmosphere and this difference effected the readings. Another variation in the readings was caused by playing the gas detecting robot at various distances from the gas`s origin this also caused variation in the readings but during this test it was also observed that the location of the gas detecting robot plays a vital role i.e. the greater is the distance from the main gas source, the reaction time of the gas detecting robot was lowered [14].

During experimentation it was noticed that the most accurate reading was taken when the air was most clean so the atmosphere should also be kept in mind while placing this robot in industry and for different atmosphere different amendments should be done in the robot.

As stated above that it is just a first stage robot and it will be improved further according to the research paper, so the circuit of this protype first stage robot consist of a Arduino mega that runs on $5 \mathrm{v}$, two de motors that also run on $5 \mathrm{v}$, a motor drive to run both the dc motors and a gas sensor that will be able to detect the leakage of certain gases.

At first the robot starts to run in the forward direction it keeps moving forward until a leakage pf certain gas is detected by the gas sensor that were placed in the front of the robot. The gas sensor on detecting a gas leakage sends a signal to the Arduino that a leakage has been detected, aa soon as the Arduino gets the signal from the gas sensor about the leakage of the gases it sends a signal to the motor driver that is connected to the $2 \mathrm{dc}$ motors to stop the dc motors as there was a gas leakage detected.

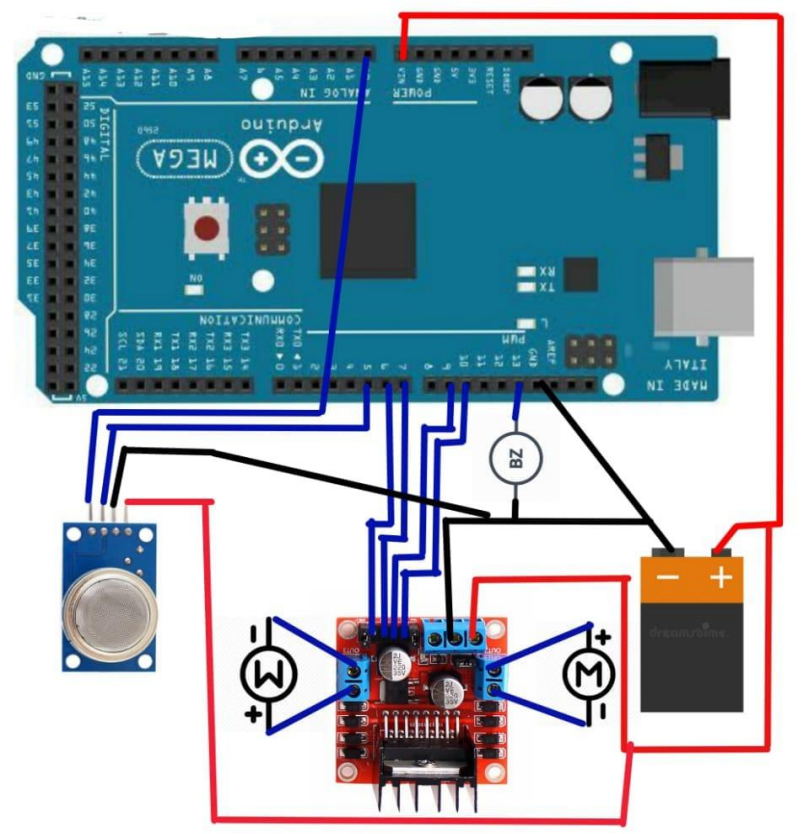

\section{Fig. 5. CIRCUIT}

To test the research, we decided to make a protype/first stage robot with an aim that the robot will be able to detect leaking gas with the help of the gas sensor.

The protype robot we decided to make comprised of a Arduino, 2 dc motors, a dc motor driver and a gas sensor to detect gas, the $2 \mathrm{dc}$ motors were of $5 \mathrm{v}$ and the operative voltage of the Arduino is also 5v so we opted to run all these things including the gas sensor with an external power supply of $9 \mathrm{v}$.

The robot initially starts to move forward until the gas sensor placed in the front of the robot detects a leaking of gas, as soon as the gas sensor detects a gas leakage it stops the robot.

So this was a prototype robot that could be further improved and made better according to the research and it can be converted directly to IOT based robot that sends signals to devices when the gas leakage is detected and mane more improvement caj be done in this robot as it is just a prototype. 


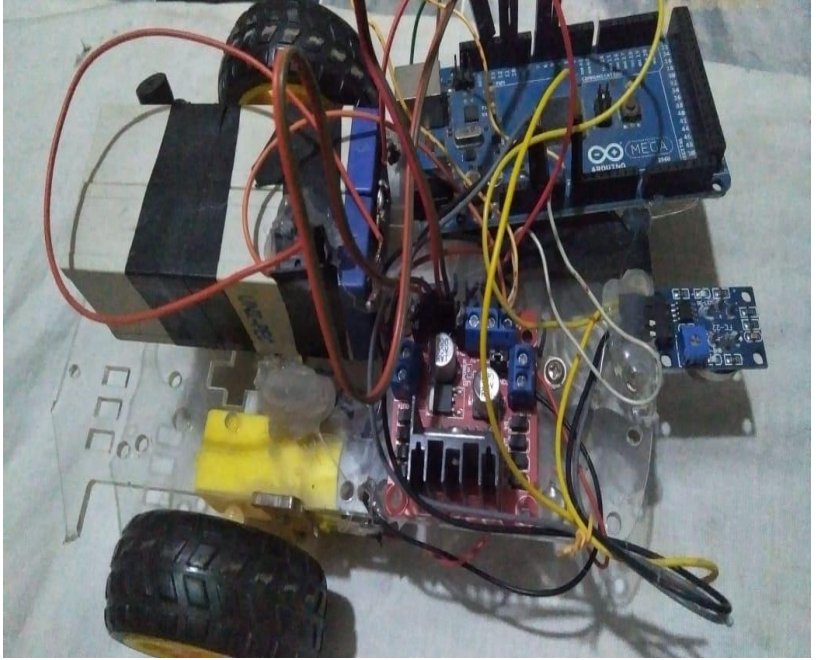

Fig. 6. ROBOT

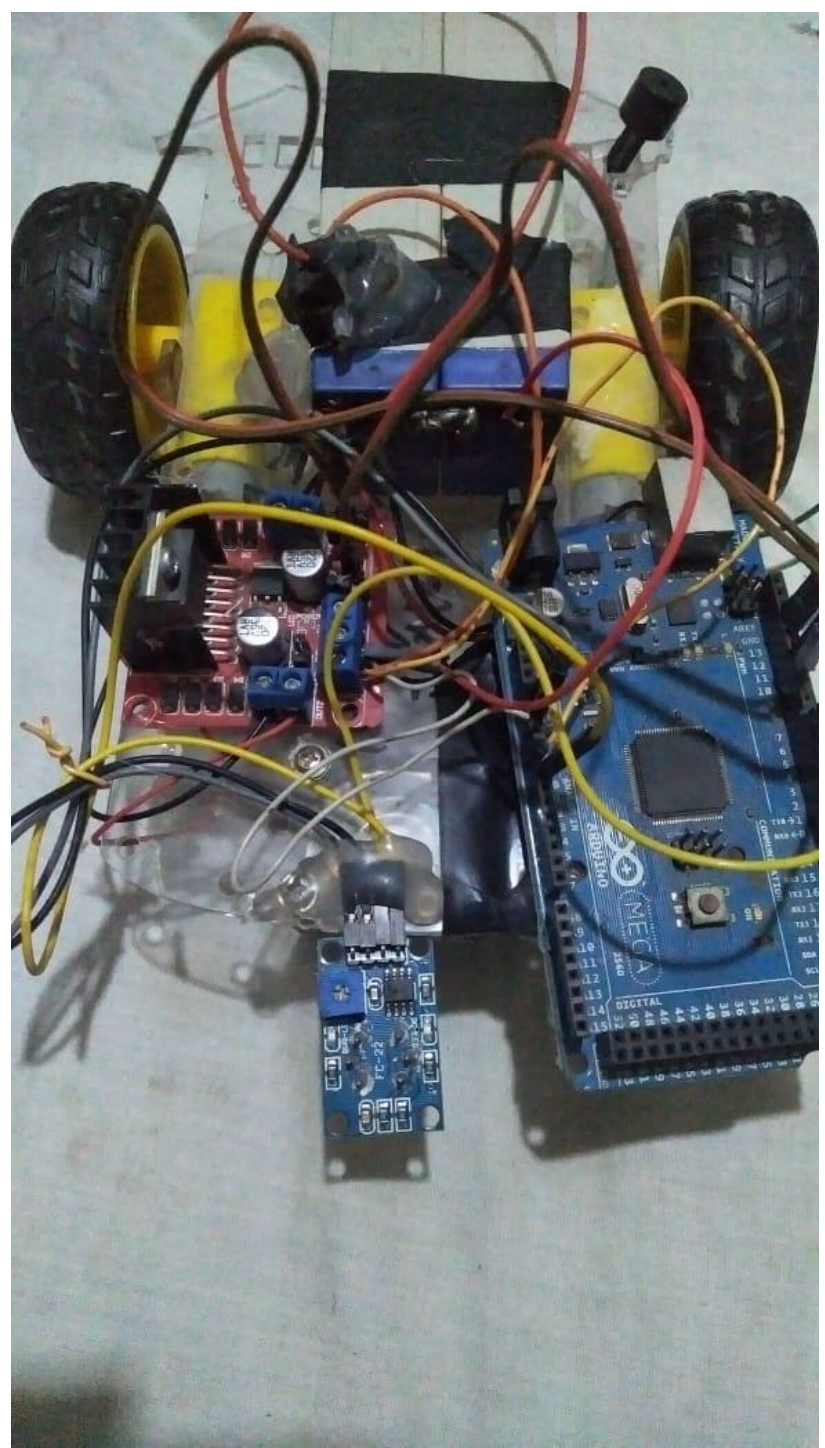

Fig.7 . ROBOT

\section{Conclusion}

To sum up, this robot is built with specific sensors and machinery to reduce the risk of any unwanted hazard's occurrences by leakage of toxic gases which are commonly used in homes, office and industrial works. This research also examined the existing work on this technology and attempted to improve the efficiency of device and make it installable at every place to get world safe and sound of accidents by gas leakages.

\section{References}

[1] T. Younas, M. A. Akbar, I. Motan, Y. Ali, M. Atif, ICETAS, 1-6 (2017)

[2] N. Bano, M. Aziz, U. Ghani, M. Taha, H. Ahmed, IOP Conference Series: Mat. Sci. \& Engr., 417,1, $012032(2018)$

[3] N. P. Aung, M. M. M. Wai, L. L. Htay, IJTSRD, 3, 5 (2019)

[4] L. Z. Ya, W. Z. dong, C. Rong, W. X. Feng,

Wu., ICIEA, 159 - 161 (2008)

[5] H. H. Yan, R. Yusnita, Design and Development of Gas Leakage Monitoring System using Arduino and ZigBee, (EESCI 2014)

[6] C. Pei-jiang, J. Xue-hua, IEEE Pacific-Asia

Workshop on Computational Intelligence and Industrial Application, 1, 678-681 (2008)

[7] R. C. Pandey, M. Verma, L. K. Sahu, IJEDR, 5, 2 , 2135-2137 (2017)

[8] A. K. Kannappan, H. Prasad, IJEDR, 5, 2, 17621768 (2017)

[9] J. Tisa, J. Lepika, J. Nedumaan, Int. Jour. of Comp. App., 178. 1-3 (2019)

[10] M. Z. Ghazali, N. M. Noor, N. Ramly, S. Putit, Procedia Engineering, 41, 1190-1196(2012)

[11] wikimedia. 2020. Raspberry Pi. [ONLINE]

Available at:

https://commons.wikimedia.org/wiki/File:Raspberry_Pi

4_Model_B_-_Side.jpg. [Accessed 20 April 2020].

[12] Circuit Pk. 2020. MQ6 sensor. [ONLINE] Available at: https://circuit.pk/product/219. [Accessed 20 April 2020].

[13] P. K. M. Lakshmi, P.S.G. A. Sri, P. G. Krishna, IJITEE, 8, 6 (2019)

U. Awasthi, N. R. Chauhan, JMSME, 3, 4, 262-267(2016) 